\title{
A NOVEL APPROACH OF ADAPTIVE SOCIALLY AWARE ROUTING ALGORITHM IN DELAY TOLERANT NETWORKS
}

\author{
Ying-Chiang Cho
}

Original scientific pape

Under the delay tolerant network, high mobility of the node and the transmission distance make it impossible to keep a durable and regular data routing path between two nodes. As a result, such failure affects the function of packet routing mechanism. Therefore, in order to improve the efficacy of data transmission, conventional delay tolerant routing adopts more packet replication, or makes opportunistic on-line with encountered nodes and selects advantageous ones to increase packet arrival rate or reduce transmission delay rate. However, excessive increase of packet replication or excessive records for selected transmission nodes may result in internet traffic or a waste of resources. It may also lead to a lower level of the routing protocol or a complicated operation. Thus, conventional delay tolerant routing is not satisfying. This research intends to design an optimized forward strategy on the basis of different routing algorithm. This strategy is based on adaptive socially aware and aims at improving solving problems of delay tolerant networks routing.

Keywords: Delay Tolerant Networks; routing algorithm; forward strategy; socially aware

Novi pristup adaptivnog društveno svjesnog algoritma za prijenos podataka u mrežama koje dopuštaju zastoj

Izvorni znanstveni članak U mreži koja tolerira zastoj, ograničenoj visokom mobilnošću čvora i prijenosnom udaljenosti, teško je održati trajnu i redovitu putanju prijenosa podataka (ruting) između dva čvora. Zbog toga, ovakvo zatajenje utječe na funkciju mehanizma za prenošenje paketa. Stoga, da bi se povećala učinkovitost prijenosa podataka, uobičajeni ruting koji tolerira zastoj prihvaća više replikacija paketa ili stvara oportunistički on-line s čvorovima na koje nailazi te izabire povoljnije kako bi ubrzao dolazak paketa ili smanjio veličinu zastoja u prijenosu. Međutim, prekomjerno povećanje replikacija paketa ili prevelik broj zapisa za izabrane čvorove prijenosa može rezultirati prometom na internetu ili gubitkom resursa. Može također dovesti do niže razine ruting protokola ili složene operacije. Prema tome, uobičajeni ruting koji tolerira zastoj ne zadovoljava. Cilj je ovoga istraživanja projektirati optimiziranu naprednu strategiju na temelju različitog ruting algoritma. Ta se strategija zasniva na adaptivnoj društvenoj osviještenosti i teži poboljšanju rješavanja problema prijenosa podataka u mrežama koje dopuštaju zastoj.

Ključne riječi: društveno osviješten; mreže koje dopuštaju zastoj; napredna strategija; ruting algoritam

\section{Introduction}

DTN (Delay Tolerant Network) is one of the focuses of wireless network in recent years [1, 4]. DTN nodes should be capable of cache. Information that cannot be transmitted in time can be placed in the buffer, waiting for transmission when conditions are ripe. Tolerance is needed in the internet to the long-time delay of information before transmitted and the packet loss rate. The transmission of DTN nodes is store-carry-forward, which means when a node meets another proper node, it can send data until the destination node or TTL (Time to live) becomes invalid.

As the link between nodes is unstable and the net topology is changeful, there are many uncertainties of routing transmission. Common routing protocols of wireless network cannot meet the need of DTN. This research proposes Adaptive Socially Aware Routing Algorithm to solve problems such as routing transmission, copy control, message priority, queuing management in DTN, and optimize forward strategy by taking the advantage of social aware of DTN nodes.

\section{DTN routing classification and assessment 2.1 Classification of DTN routing protocol}

- By differences of the network structure, routing protocol can be classified as deep space network protocol, underwater network protocol, wild social network protocol, basic social network protocol, etc.

- By the selection of routing target, routing protocol can be classified as protocol with the maximum delivery probability, the minimum cost of a copy, the minimum average delay, the minimum average number of hops, etc.

- By differences of routing mechanism, routing protocol can be classified as protocol with or without prior knowledge.

- By the number of copies, routing protocol can be classified as single copy or multiple copies.

- By whether there is infrastructure or not, routing protocol can be classified as protocol with or without infrastructure.

\subsection{Assessment of DTN routing protocol}

The three indicators, delivery probability, average latency and overhead ratio, are commonly used to measure the overall performance of the routing protocol, which can reflect the performance of routing protocols in DTN network. How to design an algorithm with three ideal indicators is a difficult problem facing in front of researchers.

\section{DTN routing algorithm \\ 3.1 Routing protocol based on zero knowledge}

In order to maximize message delivery probability, minimize message transmission delay and maximize the use of internet resources, Vahdat and Becker propose an epidemic routing algorithm [8] based on partly linked AD HOC network. The key idea is that message can be transmitted to destination node within the shortest time when two nodes meet and exchange all message. Lindgren and some others propose a prophet routing algorithm 
[9]. They think that in DTN, it is necessary to introduce delivery probability for helping with routing decision. There are three steps:

$P(x, y)=P(x, y)_{\text {old }}+\left[1-P(x, y)_{\text {old }}\right] P_{\text {init }}$,

$P(x, y)=P(x, y)_{\text {old }} \times \varepsilon^{l}$,

$P(m, p)=$

$=P(m, p)_{\mathrm{old}}+\sigma\left[1-P(m, p)_{\mathrm{old}}\right] P(m, n) P(n, p)$.

$P_{\text {init }} \in[0,1]$ is the initial constant, $\varepsilon$ is the attenuation constant, $l$ is the number of periods since nodes last met. $\sigma \in[0,1]$ is for quantifying influence probability of transmission. If two nodes still fail to meet in a certain period of time, then they are labelled as a low probability of meeting. When the node that carries message meets a new node, then compare the arrival probability of different destination nodes. If the delivery probability of the new node is higher than that of the node that carries message, then message shall be transmitted. Otherwise, they remain still. Spyropoulos and the other propose sprayand-wait routing algorithm [10]. In the phase of spraying, the source node transmits message in a way close to epidemic algorithm. In the phase of waiting, the source node only transmits message when it meets the destination node. Zhao and some others propose a ferry-node routing algorithm [11]. It includes two different frameworks: one is NIMF (node-initiated message ferrying scheme), the other is FIMF (ferry-initiated message ferrying scheme). In NIMF, ferry node moves in accordance with predetermined orbit. When a node has a message to transmit, it moves to ferry node. In FIMF, such node sends request to the ferry node and the latter responds to the request by changing moving orbit, when a node has a message to transmit.

\subsection{Routing protocol based on knowledge}

Linear programming algorithm of DTN routing can be divided into zero-knowledge, partial-knowledge and complete-knowledge [12]. Due to limited prior knowledge, the routing protocol based on complete knowledge only has the value as a reference to algorithm comparison. Some typical comparisons include: FC (First Contact), MED (Minimum Expected Delay), ED (Earliest Delivery), EDLQ (Earliest Delivery with Local Queue), EDAQ (Earliest Delivery with All Queue), LP (Linear Program) and other protocols. Burgess and some others propose message-queuing-management routing algorithm [14], aiming at a lack of neighbouring information and a brevity of contacting time between nodes.

\subsection{Other routing protocol}

Nelson and some others propose a contacting - DTN routing algorithm [15]. They think that the future meeting probability of different nodes can be predicted by past contacting information. Every node needs to preserve past meeting frequency $f_{\text {ever }}$ and the times $\left(n_{\text {current }}\right)$ of meeting within current period of time. The algorithm of $f_{\text {ever }}$ is as follows:

$f_{\text {ever }} \leftarrow \alpha n_{\text {current }}+(1-\alpha) f_{\text {ever }}$.
When node $M$ meets node $N, M$ transmits $\frac{a_{i} n_{\text {current }}^{N}}{n_{\text {current }}^{M}+n_{\text {current }}^{N}}$ copies of message to node $N, a_{i}$ refers to number of copies of message.

To reduce the huge cost of copy overhead of epidemic routing algorithm, Liu and $\mathrm{Wu}$ propose a bestprobability-transmission routing algorithm [16]. When node $\mathrm{i}$ meets node $\mathrm{j}$, the node only transmits message to nodes with higher delivery probability $P_{i, d, K, T_{r}}(d$ refers to destination node, $k$ refers to remaining message hops, $T_{r}$ refers to TTL), that is to judge whether it meets:

$1-\left(1-P_{i, d, K-1, T_{r}-1}\right) \times\left(1-P_{j, d, K-1, T_{r}-1}\right) \geq$

$\geq P_{i, d, K, T_{r}-1}$.

If it does, then it transmits message, otherwise remains still.

Balasubramanian and some others propose a routing algorithm that regards routing strategy as resource distribution. 1) Nodes exchange message with each other to calculate the efficacy of message. 2) If the neighbour node is the destination node, then message shall be transmitted. 3) Create related copies based on different utility functions. 4) The process ends with all message getting copies and being transmitted. The minimum average delay is referred to as utility function $\Phi_{i}$ :

$\Phi_{i}=-t_{\text {delay }}(i)$.

$t_{\text {delay }}(i)$ refers to delay of message. In order to reduce the number of messages that miss the period of time, utility function is defined as:

$\Phi_{i}=$
$=\left\{\begin{array}{cc}P\left\{a(i)<t_{\text {live }}(i)-t_{\text {send }}(i)\right\}, & t_{\text {live }}(i)>t_{\text {send }}(i) \\ 0, & \text { otherwise }\end{array}\right.$.

where $t_{\text {live }}(i)$ refers to living time of message, $t_{\text {send }}(i)$ refers to sending time. In order to minimize the maximum delay, the utility function is defined as:

$\Phi_{i}=\left\{\begin{array}{cc}-t_{\text {delay }}(i), & t_{\text {delay }}(i) \geq t_{\text {delay }}(j) \quad \forall j \in K \\ 0, & \text { otherwise }\end{array}\right.$.

$K$ refers to all messages in the buffer.

\section{Social aware of DTN}

Adaptive social aware in DTN includes: the distribution of community, and the calculation of centrality, the calculation of similarity and the confirmation of friend relationship. More often than not, people or equipment operated by people serve as wireless equipment in DTN. Thus, understanding social relations in DTN is significant for improving the performance of routing algorithm [17].

Common standards for centrality include: degree centrality, closeness centrality, stress centrality, betweenness centrality, graph centrality, etc. Standards for similarity are measured by the number of public neighbours of different individuals in social network. They are also calculated by interest, age, occupation and geographical distri- 
bution. The closeness of two nodes can be illuminated by friend relationship.

\section{Routing algorithm based on social aware}

Hui and Crowcroft propose a Pocket Switched Networks (PSN) [19] routing algorithm based on social tag. They set up a tag for every node to show the community to which the node belongs. The source node shall transmit message to the destination node or to other nodes in the same community to which the destination node belongs.

Hui and some others propose a Bubble Rap routing algorithm [20]. The whole system is in the phase of transmission before message arrives at the community to which the destination node belongs. The whole system enters the phase of partial-transmission when message arrives at the community to which the destination node belongs until they are transmitted to the destination node.

Daly and Haahr propose a SimBet routing algorithm [21] based on social relationship. Estimate betweenness centrality $\mathrm{Bet}_{n}$ on the basis of $\mathrm{n}$ nodes in the local neighbourhood, and take the number of common neighbours between the current node $a$ and destination node $\mathrm{s}$ as its similarity index $\operatorname{Sim}_{a}(s)$. When node $a$ (which carries message for destination node $s$ ) meets node $b$, node $a$ shall calculate related betweenness centrality and similarity index:

$\overline{\operatorname{Sim}}_{a}=\frac{\operatorname{Sim}_{a}(s)}{\operatorname{Sim}_{a}(s)+\operatorname{Sim}_{b}(s)}$,

$\overline{\operatorname{Bet}}_{a}=\frac{\operatorname{Bet}_{a}}{\operatorname{Bet}_{a}+\operatorname{Bet}_{b}}$.

Then calculate the efficacy of node a:

$\overline{\operatorname{SimBet}}_{a}(\mathrm{~s})=\mu \overline{\operatorname{Sim}}_{a}(s)+(1-\mu) \overline{\mathrm{Bet}}_{a}$.

$\mu$ is an adjustable parameter. If $\overline{\operatorname{SimBet}}_{b}(s)>$ $\overline{\operatorname{SimBet}}_{a}(s)$, a shall transmit message to $b$. Otherwise, message shall stay with $a$.

\subsection{Routing algorithm based on friend relationship}

Bulut and some others propose a routing algorithm in which a node transmits message through friend relationship [22]. It introduces Social Pressures Metric to quantify friend relationship, which is SPM between node $\mathrm{m}$ and node $n$ :

$\mathrm{SPM}_{m, n}=\frac{\int_{0}^{T} v(t) \mathrm{d} t}{T}$

$v(t)$ refers to the period of time between the first time that node $m$ meets node $n$ after time $t, T$ refers to time cycle. If node $m$ carries message to node $n$ in every time cycle, then SPM describes average transmission delay. Define link weight as $\psi_{m, n}=\frac{1}{S P M_{m, n}}$. Suppose $\psi_{m, n}$ refer to the strength of friend relationship between $m$ and $n$. By $\psi_{m, n}$, a node can construct its own friend community in every $T$. When $m$ (which carries message for destination node $p$ ) meets $n$, if and only if $n$ and $p$ are in the same friend community in the current cycle and $n$ is a better friend for $p$ than $m$ is, $m$ shall transmit message to $n$.

\subsection{Routing algorithm based on other social relationship}

Mei and some others propose PSN network routing algorithm (SANE) [23] that is based on common interest. It parallels a person's $x$ interests to $l$ dimensional tor $R_{x}$, and defines similarity of interest between two individuals as

$\Omega\left(R_{x}, R_{y}\right)=\cos \left(\angle R_{x}, R_{y}\right)=\frac{R_{x} R_{y}}{\left\|R_{x}\right\|\left\|R_{y}\right\|}$

If and only if similarity of interest between message $p$ and node $x$ is higher than the threshold can $p$ be transmitted to $x$

Gao and Cao propose a user-oriented data distribution approach [24]. It takes social centrality and users' interest into consideration and redefines centrality as the estimated number of people that the node takes interest in in the rest time of data distribution. Based on this changeful centrality, relay selection of the routing can guarantee a better data distribution of the new relay selection to those who are interested in it. Fabbri and Verdone propose a social-nature routing strategy [25].

They think that if a node often contacts with different nodes, this node is deemed as a good transmitter, and it only transmits message to nodes with the highest social nature. They also propose a SOSIM routing algorithm using historical contacting information as the similarity index to increase accuracy and introduces transmission agency to increase efficiency. Wu and Wang propose a multi-path transmission DTN routing algorithm [27] focuses on the internal social characteristics. In the phase of extracting social characteristics, entropy is used to construct a characteristic space $\left(S_{1}, S_{2}, \ldots, S_{k}\right)$ from $\mathrm{N}$ most representative social characteristics, with $S_{i}$ standing for one of the social characteristics. When there are $m$ nodes and $\mathrm{n}$ characteristics in the internet, quantify character information:

$I\left(S_{j}\right)=-\sum_{i=1}^{m} p\left(a_{i}\right) \log _{2} p\left(a_{i}\right), \quad(j=1,2, \ldots, k)$.

Thus, routing decision becomes a process of matching characteristics. They also propose Mobile Opportunistic Social Networks message distribution approach [27, 28] based on social relationship:

$\xi_{m n}=u E_{m n}+\frac{v}{1+S_{m n}}$

$E_{m n}$ stands for times of standardization connections between node m and node n. $S_{m n}$ stands for weight of social attribution between two nodes. $\mu$ and $v$ stand for weight of contacting information and social attribution information respectively $(u+v=1)$. We first analyse MIT reality mining data group [29], then conduct data distribution protocol based on social relationship. 


\section{Adaptive socially aware routing algorithm}

The routing decision of DTN mainly faces the following problems: 1) Whether to transmit message to nodes when they meet; 2) What message can be transmitted when nodes meet; 3) How to effectively control the number of message copies; 4) How to confirm the priority of message; 5) How to manage message-queuing. To solve the abovementioned problems, this research proposes Adaptive Socially Aware Routing Algorithm. Its core idea is: firstly use community distribution algorithm to divide the internet into different societies, then measure the importance of the node in the internet by whole centrality and that in the local community by partial centrality. Confirm routing transmission strategy by social information and centrality.

We employ dichotomy message-copy-controlling mechanism to reduce the copy overhead. Grant priority to message by utility function and provide rank for opportunistic transmission and losing message. We use proportional - integral - derivative for queuing management to avoid the overflow and the internet traffic. As ASARA adopts strict message transmission strategy, message delay is superior to other algorithms. Community distribution is adopted as many research results indicate that the contacting frequency of internal nodes in the same community is much higher than meeting rate with nodes in other societies. It is reasonable to take nodes that are in the same community with the destination node as the relay node. Though single community distribution may improve the efficiency of algorithm [18], this research finds out that the result is limited and needs other strategies for further improvement. This research adopts centrality to measure the importance of nodes, that is, to take the number of neighbours of a node as the centrality. The whole centrality measures the importance of node in the internet. It equals to the number of all neighbour nodes. Partial centrality measures the importance of node in local community. It equals to the number of all neighbour nodes in local community. The concept of neighbour is based on accumulated contacting time that exceeds a certain threshold. Part of DTN routing algorithm adopts contacting frequency as an important standard to measure nodes. However, large amount of researches show that in DTN, some nodes establish frequent connection with other nodes but the contacting time period is hard to maintain so that they cannot meet the time period for opportunistic transmission. If contacting frequency serves as a standard, then similar nodes may be disadvantaged to the performance of the protocol. The existing messagecopy-controlling mechanism has its weaknesses, for example, complicated calculation, wasting calculation resources of nodes, the result of controlling links to the net environment that varies with DTN, which makes it hard to estimate copy overhead.

Because of this, the present research proposes a dichotomy message-copy-controlling mechanism: Messages are granted with original copy number (A power of 2 ). The copy is divided into 2 when transmitted every time, one is to the source node, the other is to the relay node. As DTN is linked by opportunistic transmission which is easy to be disconnected, researchers propose different optimized strategies to improve delivery probability, average delay, number of copies, average hops and other indices to increase message of the internet performance. As DTN has a low delivery probability on which all applications rely, this research adopts delivery probability as the target function. It proposes a new utility function aimed at movement regularity of nodes of social aware DTN to calculate the weight of every message, and confirm the priority of message transmission in accordance with the weight, and provide support for message transmission and losing message. DTN node's cache is strictly limited. As a result, message queuing management is ignored. The most common approach is to lose message that stays the longest time in cashes when there is overflow. But this approach fails to take into consideration the effect on overall delivery rate of the internet by losing message. Therefore, this research proposes a proportional - integral - derivative controller to control queue length. When the length exceeds a certain threshold, message with the lowest efficacy shall be missing.

\subsection{Transmission strategy of community distribution \& distributed community-discovering routing algorithm}

This research thinks that understanding social information of the destination node can help the transmitting node to select the proper relay node. Given advantages of different routing transmission strategy, this research proposes an improved BUBBLE RAP strategy. The main ideas are as follows: divide the internet into societies with different scales by community distribution algorithm; use whole centrality to measure the importance of nodes in the internet; use partial centrality to measure the importance of nodes in local community. As meeting frequency of nodes in the same community is higher than that with nodes in other societies, the partial centrality is superior to the whole centrality.

During the experiment, many messages are transmitted in nodes with more or less similar centrality. This takes up rooms of bandwidths of nodes and increases energy consumption. Therefore, in the improved routing transmission strategy, nodes with different centrality can be transmitted to each other. When a node meets its destination node, message should be transmitted initially. Thus, this is a prior judgment. With these improvements, routing transmission strategy presents good performance in the experiment. When node $m$ (which carries message for destination node $p$ ) builds up opportunistic connection with node $n, m$ may encounter the following situations:

1) If $n$ equals $p$, then transmit.

2) If $m, n, p$ are in the same community, and if the partial centrality of $n$ exceeds $m$ to some extent, then $m$ shall transmit.

3) If $m, n, p$, are in the same community, but $m$ is close to $n$ in terms of partial centrality, then $m$ shall not transmit.

4) If $n$ and $p$ are in the same community, and $m$ is in another community, then $m$ shall transmit.

5) If $m$ and $p$ are in the same community, and $n$ is in another community, then $m$ shall not transmit.

6) If $m$ and $n$ are in the same community where $p$ is in, but if the partial centrality of $n$ exceeds $m$ to some extent, then $m$ shall transmit. 
7) If $m$ and $n$ are in the same community where $p$ is in, but $m$ is close to $n$ in terms of partial centrality, then $m$ shall transmit.

The researchers propose many centralized community-discovering algorithms [30, 31]. They take static analysis on movement obit of data group, and try to find out internal structure and regularity to design effective transmission strategy, safety measures and internet applications. In DTN, no node can obtain information about the whole internet. Thus a node needs to judge its community by historical contacting information.

Many networks do not have independent social structures. On the contrary, they consist of many interdependent societies. A community is to some extent viewed as a group of inter-connected small full coupling networks, which is called clique. Ad-clique refers to $d$ nodes of full coupling networks. If two d-cliques have $d-1$ common nodes, then these two d-cliques are neighbours. If a dclique can arrive at another d-clique by many other cliques, then these two d-cliques are inter-connected [30]. One person may belong to many societies and d-clique algorithm can meet the demand of this nature. In DTN, moving nodes need to find out societies they belong to and cannot rely on centre node in conventional internet. In this case the conventional d-clique communitydiscovering algorithm is not proper.

This research adopts distributed d-clique algorithm as community distribution algorithm [34]. Experiments show that the overhead of d-clique is moderate with good results and suitable for DTN community-discovering. Distributed d-clique algorithm is defined as follows: Familiar Set $S_{F}$ refers to every node and can keep a record of contacting time period of meeting nodes. If accumulated contacting time period exceeds the threshold, it shall be added to $S_{F}$.

According to the definition, for a node $n_{i}$, its familiar set is $S_{F}^{i}$. Meanwhile, a node can obtain partial information through the familiar set of other nodes, which is the estimation of the familiar set of other nodes. Information group from the familiar set of other nodes for node $n_{j}$ can be represented by $\tilde{S}_{F}^{j}$. The local community of a node includes all nodes (direct neighbours) in the familiar set of this node and other nodes discovered by community-discovering algorithm (specific standards will be detailed in the following context).

Given asynchrony of time, different nodes in the same community may appear in two communities. This deviation brought by frequent change in DTN is unavoidable. Neighbour set is represented by $S_{N}$. The neighbour net of local community $L C_{0}$ can be represented by $S_{N}^{0}$, which consists of such nodes: every node is not in $L C_{0}$, but every node is linked with one or many nodes in $L C_{0}$, that is

$S_{N}^{0}=\left\{n_{i} \mid n_{i} \in \cup_{n_{j} \in L C_{0}} S_{F}^{j} \backslash L C_{0}\right\}$

Close-topology set $F S_{0} L C_{i}$ refers to all familiar sets of nodes in $L C_{0}$ are in a close set. The basic steps of algorithm are as follows: When a moving node $n_{0}$ starts its community-discovering process, $n_{0}{ }^{\prime} s L C_{0}$ only has $n_{0}$; when $n_{0}$ meets other node $n_{i}$, they exchange partial mes- sage. $n_{0}$ shall decide: 1$)$ Whether to add $n_{i}$ to $S_{F}^{0}$ or $L C_{0}$; 2) Put $L C_{0}$ and $L C_{i}$ together completely or partially. The specific steps are shown in Fig. 1.

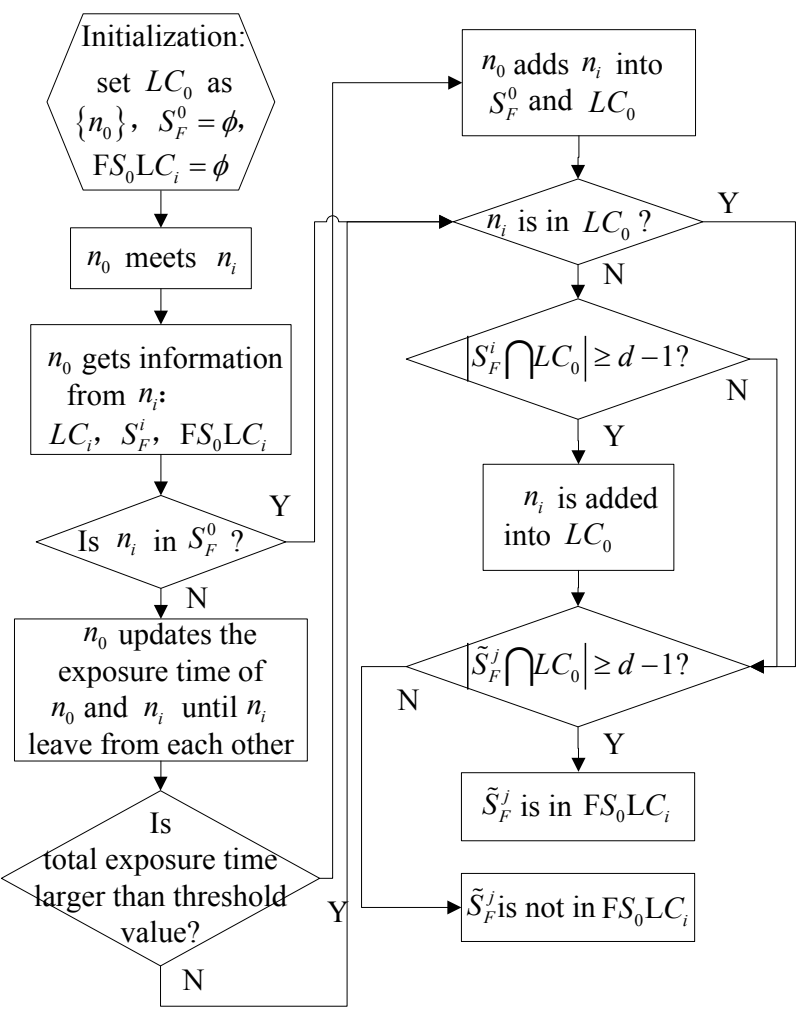

Figure 1 Algorithm flow chart

\subsection{Calculation of centrality \& copy-control strategy}

This research uses centrality to measure the importance of nodes in DTN and proposes two calculation approaches: degree centrality and single-window centrality. In the graph, the simplest concept is centrality that is defined as the number of connections of a node. In social network, centrality is defined as the number of relations of a node. In the directed network (in which the relation between nodes is directed), centrality is divided into two categories: degree and out-degree. Degree refers to the number of relations directed to one node. Out-degree refers to the number of relations directed to other nodes from one node. In DTN, opportunistic connection between nodes is double-sided. And only with double-sided connection can the connection be regarded as "being established". This research selects undirected graph to establish relations between nodes. In fact, the calculation of centrality in the graph needs all information of internet topology. However, in DTN, it is hard to obtain key information for this calculation. Therefore, how to estimate the single-window centrality of nodes remains to be solved. In DTN, researchers use contacting frequency of nodes as the standard of centrality. The result indicates that some nodes contact frequently with other nodes but the contacting period is short. If contacting frequency is made as the standard of centrality, then similar nodes may result in poor performance of the protocol. Thus this research obtains an accumulated contacting period of one node with all the others by counting contacting histories in DTN and takes it as neighbour standard of this node. 
By calculating the number of neighbours of a node in the whole internet and local community, we can obtain the whole centrality and partial centrality. This calculation adapts to common situation in the social network, that is, if someone contacts frequently with others and the contacting period is long, then this person is welcomed in the internet and obviously he is proper to transmit message, for example, tellers and waiters. In the pretext, the centrality is the number of neighbours of a node. But even so, there is some limitation as in DTN, the contacting of a node changes with time: in some periods, nodes are active and contact more frequently with others and have longer contacting period, so nodes at this time are proper to transmit message; however in some periods, the centrality of a node could be low. Another algorithm is to calculate the centrality of the nearest time window [20]. This approach takes the number of neighbours of a certain period as the standard of centrality and has achieved good result by taking time into consideration. To control copies in DTN, researchers adopt several copy-control strategies such as optimized stopping theory and probability estimation. But it requires a large amount of calculation and is easy to be affected by the environment. Inspired by cell proliferation, this research proposes a relatively simple yet stable dichotomy message-copy-controlling mechanism: grant an original number of copies before creating message, the number of which is the power of 2 (the power is not zero); when a node meets a proper relay node, it transmits message to relay nodes. The number of copies got by relay node is $1 / 2$ of that of the current node, and the number reduces to $1 / 2$ of the original number of this node and so on. When the number of copies of message is 1 , the node shall not transmit message to relay nodes, yet it does when it meets the destination node, until the TTL turns to zero. As a result, the number of copies is controlled under a reasonable range. In the experiment, given the range of 36-98 of nodes in data set, there are 16 original copies. The result indicates that this strategy works well and lowers the copy overhead without grabbing the calculation resources from nodes.

\subsection{Message-dispatching strategy of DTN routing}

In social aware routing algorithm, this research first considers the effects placed on the routing by movement model. In DTN, only by two nodes move into the realm of each other can message be transmitted. Therefore, meeting time is an important part of message transmission delay. The result shows that a movement model is adapted to exponential distribution. In a relaxed network (also applies to DTN), number of samples can be estimated by stable meeting time or the meeting time of nodes adapts to exponential distribution [37]. Meeting time of nodes for optimizing routing transmission strategy becomes a focus of researchers [36]. The basis hypotheses are as follows:

1) Meeting time is adapted to exponential distribution

2) Movements of nodes are independent from each other

3) Movements of nodes are the same, that is, all nodes have the same meeting frequency $f$.

The first hypothesis is proved in related literature. In this research, movements of nodes in DTN are independent from each other, that is, movement of the first node does not influence on others. Under the second hypothesis, the focus of the research is social network with the same characteristics of movement and no other complicated movement models are considered. In later experiments, opportunistic contacting of real data set is in line with the previous three hypotheses. Given situations when node $m$ meets node $n$.

Table 1 Variables of the utility function

\begin{tabular}{|c|l|}
\hline Variables & \multicolumn{1}{c|}{ Function } \\
\hline$K$ & Number of nodes in the internet \\
\hline$L(t)$ & $\begin{array}{l}\text { Number of message in the internet at time } \\
T \text { (copies are not counted) }\end{array}$ \\
\hline$T T L_{m}$ & Original living time of message $m$ \\
\hline$R_{m}$ & Rest living time of message $m$ \\
\hline$T_{m}=T T L_{m}-R_{m}$ & $\begin{array}{l}\text { Death time of } m \text {, period since the original } \\
\text { living time of } m\end{array}$ \\
\hline$N_{m}\left(T_{m}\right)$ & Number of copies after time $T_{m}$ \\
\hline$M_{m}\left(T_{m}\right)$ & $\begin{array}{l}\text { Number of nodes that transmit message } m \\
\text { after time } T_{m}\end{array}$ \\
\hline$f$ & $\begin{array}{l}\text { Contacting frequency of two nodes; } f= \\
\frac{1}{E[H]}, E[H] \text { refers to average contacting } \\
\text { time }\end{array}$ \\
\hline
\end{tabular}

In the process of opportunistic transmission, bandwidth of nodes and the cache in DTN are limited in many ways, one of which is message-dispatching strategy. When the whole centrality of $m$ is higher than that of $n, m$ needs to transmit message to $n$. Due to limited bandwidth and in order to reach the optimized delivery rate in the internet, we should consider the priority of message in the cache. In opportunistic transmission, the connection can be stopped at any time. Thus the priority of message matters a lot to transmission. In addition, when the cache for $\mathrm{n}$ is full, which message to lose when there is a new one to receive? Reasonable selection prevents the delivery rate from being influenced or only makes the delivery rate influenced slightly. In routing algorithm of DTN, the most important standard is the delivery rate. A higher delivery rate means a more reliable protocol to upper-layer applications. Therefore, in the designing of protocol, the delivery rate is a primary standard. To optimize the overall delivery rate of the internet, this research proposes a utility function for every message, the value of which is taken as standards for nodes for message queuing management and message dispatching strategy.

Lemma 1: Suppose there are $L$ different messages in DTN; for every message $\mathrm{m}$, death time is $T_{m}$. For every $m \in[1, L]$, take $N_{m}\left(T_{m}\right)$ as the number of nodes that have copies of m. Take $M_{m}\left(T_{m}\right)$ as the number of nodes that transmit $\mathrm{m}$ since messages are created. The following utility function for every $\mathrm{m}$ is made to reach the highest delivery rate in DTN:

$\mathcal{E}_{m}(\mathrm{DR})=\left(1-\frac{M_{m}\left(T_{m}\right)}{K-1}\right) f R_{m} \exp \left(-f N_{m}\left(T_{m}\right) R_{m}\right)$.

Prove: the copy of $\mathrm{m}$ cannot be transmitted, if and only if the meeting time with the destination node is longer than $R_{m} \cdot R_{m}$ refers to the rest living time of sage $\left(R_{m}=T T L_{m}-T_{m}\right)$. According to the hypothesis, the time is $\exp \left(-f R_{m}\right)$. As the number of copies of $m$ is $N_{m}\left(T_{m}\right)$, and suppose message has not been transmitted, 
thus we can deduce the un-delivery rate of message (all copies of message haven't been transmitted):

$P\{m$ hasn't been transmitted|all haven't been transmitted $\}$

$=\prod_{l=1}^{N_{m}\left(T_{m}\right)} \exp \left(-\mathrm{f} R_{m}\right)=\exp \left(-f N_{m}\left(T_{m}\right) R_{m}\right)$.

Temporarily, we do not consider that $\mathrm{m}$ shall create more copies. Nor do we consider that $\mathrm{m}$ is lost in time $R_{m}$. Thus, this strategy is the most optimal in terms of time. If we consider the meeting of future nodes and the creation of message copies, then it turns out to be more complicated.

Given that there is no clear marks for a successful delivery in DTN, all nodes (including destination nodes) have the same opportunity to receive message. Thus successful delivery rate of $\mathrm{m}$ is:

$P\{m$ that has already been transmitted $\}=\frac{M_{m}\left(T_{m}\right)}{K-1}$.

Linking two formulae above: the rate of successful delivery of $\mathrm{m}$ before the living time $T T L_{m}$ expires:

$P_{m}=P\{m$ that hasn't been delivered $\} *\{1-$ $\left.\exp \left[-f N_{m}\left(T_{m}\right) R_{m}\right]\right\}+P\{\mathrm{~m}$ that has been delivered $\}=$ $\left[1-\frac{M_{m}\left(T_{i}\right)}{K-1}\right] *\left\{1-\exp \left[-f N_{m}\left(T_{m}\right) R_{m}\right]\right\}+\frac{M_{m}\left(T_{m}\right)}{K-1}$.

Thus, overall delivery rate of message in the internet at time $\mathrm{t}$ is:

$$
\begin{aligned}
& D R=\sum_{m=1}^{L(t)}\left[\left(1-\frac{M_{m}\left(T_{m}\right)}{K-1}\right)\left\{1-\exp \left[-f N_{m}\left(T_{m}\right) R_{m}\right]\right\}+\right. \\
& \left.\frac{M_{m}\left(T_{m}\right)}{K-1}\right] .
\end{aligned}
$$

When opportunistic transmission happens or when the queuing is full, nodes shall take an optimal message to copy/lose to maximize $D R$, that is, to maximize $\Delta(D R)$ :

$$
\begin{aligned}
& \Delta(D R)=\sum_{m=1}^{L(t)} \frac{\partial P_{m}}{\partial N_{m}\left(T_{m}\right)} * \Delta N_{m}\left(T_{m}\right)=\sum_{m=1}^{L(t)}[(1- \\
& \left.\left.\frac{M_{m}\left(T_{m}\right)}{K-1}\right) *\left\{1-\exp \left[-f N_{m}\left(T_{m}\right) R_{m}\right]\right\}+\frac{M_{m}\left(T_{m}\right)}{K-1}\right] .
\end{aligned}
$$

In the process of transmission, if transmit a new message $m, \Delta N_{m}\left(T_{m}\right)=+1$. When conducting message queuing management, if it is necessary to delete $\mathrm{m}$ from the cache, $\Delta N_{m}\left(T_{m}\right)=-1$. If $\Delta N_{m}\left(T_{m}\right)=0$, do nothing to the cache. Thus, there is the utility function for every message by message-queuing management and dispatching strategy:

$$
\mathcal{E}_{m}(D R)=\left(1-\frac{M_{m}\left(T_{m}\right)}{K-1}\right) f R_{m} \exp \left(-f N_{m}\left(T_{m}\right) R_{m}\right) .
$$

Then, make related decision. The value of efficacy depends on two variables of message $i\left(N_{m}\right.$ and $\left.M_{m}\right)$.

In order to maximize the overall delivery rate in the internet, this research deduces a utility function, in which the number of nodes $K$, contacting frequency between two nodes f and variables $N_{m}$ and $M_{m}$ are not fixed values. As this function directly decides the effective result of message-queuing-management, thus this research uses the number of nodes and contacting frequency from the data set for calculation. In the process of calculating, the only parameter to be estimated is two variables of message $\mathrm{m}$, $N_{m}$ and $M_{m}$. As this research introduces message-copycontrolling mechanism, thus $N_{m}=M_{m}+1$. Calculating any one of them ensures a complete utility function.

In DTN, every node can only obtain part of the contacting information, thus a node cannot get $N_{m}$ or $M_{m}$ of every message. This research proposes a wholeparameter-estimation approach based on historical information [38], which is to estimate whole parameters by statistical information, that is, estimating whole information by partial information in the internet. This research adopts an approach based on historical information to estimate $M_{m}\left(T_{m}\right)$ (the number of nodes that transmit message $\mathrm{m}$ after time $\left.T_{m}\right) . \breve{M}_{m}\left(T_{m}\right)$ refers to random variable of $M_{m}\left(T_{m}\right)$ :

$E\left(M_{m}\left(T_{m}\right)\right)=\frac{\sum_{m=1}^{n} M_{m}\left(T_{m}\right)}{n}$.

$n$ refers to the total number of messages that this node transmits. The average dying time of every message can be calculated as well:

$\widehat{T}=\frac{\sum_{m=1}^{n} T_{m}}{n}$.

The estimation value $\widehat{M_{p}\left(T_{p}\right)}$ of $M_{m}\left(T_{m}\right)$ of message $m$ is:

$\left.\widehat{M_{p}\left(T_{p}\right.}\right)=\frac{T_{m} E\left[\breve{M}_{m}\right]}{\widehat{T}}=\frac{T_{m} \cdot \sum_{m=1}^{n} M_{m}\left(T_{m}\right)}{\sum_{m=1}^{n} T_{m}}$

Thus, efficacy value of message $\mathrm{m}$ can be calculated by $\widehat{M_{p}\left(T_{p}\right)}$ and other parameters.

\subsection{Queuing length management of nodes in DTN}

In DTN nodes, cache is limited strictly. Conventional DTN nodes usually adopt first in, first out (FIFO) way to store message and lose message randomly when there is an overflow without any queuing management. However, overflow may exert great influence on the overall delivery rate when nodes transmit too much message. Thus it is necessary to manage the length of cache. This research adopts proportional - integral - derivative approach to manage queuing length:

$P(n)=P(n-1)+K_{p}[E(n)-E(n-1)]+K_{I} E(n)+$

$K_{D}[E(n)-2 E(n-1)+E(n-2)]$.

$E(n)=Q_{C U R}-Q_{R E F}, Q_{C U R}$ refers to current length of queuing. $Q_{R E F}$ refers to the standard length of queuing. Every time when a new message arrives, the node produces a random probability $P_{\text {rand }}$, if $P(n)>P_{\text {rand }}$, then loses the message that has the smallest weight in the queue. According to deviation of input, operation the function of proportional, integral and derivative and output the result. The structure of proportional, integral and derivative is flexible in accordance with the controlled object and controlling requirement. Take some part as controlling regularity, such as proportional adjustment, 
proportional - derivative adjustment, proportional - integral - derivative adjustment, etc.

As a proportional adjustment alone cannot eliminate static deviation, it is necessary to introduce integral adjustment (because the result of integral adjustment changes with the input value). Introducing proportional adjustment is for pre-adjustment because only when the input jitters will the output change. Proportional adjustment is related to deviation. Derivative adjustment is related to positive or negative of deviation. Integral adjustment is related to jitter of deviation. In the mimic system, the formula of proportional - integral - derivative is:

$P(t)=K_{p}\left[e(t)+\frac{1}{T_{I}} \int e(t) \mathrm{d} t+T_{D} \frac{\mathrm{d} e(t)}{\mathrm{d} t}\right]$.

$P(t)$ refers to input signal of the controller; $e(t)$ refers to deviation signal of the controller, the value of which equals to the difference between the controlling value and the fixed value; $K_{p}$ refers to proportional coefficient of the controller; $T_{I}$ refers to derivative time of the controller; $T_{D}$ refers to the derivative time of the controller. The controlling system should discretize the formula, and use difference equation to replace integral equation. At this moment, derivative item and integral item can be represented by:

$\int \mathrm{d} t=\sum_{j=0} E(j) \Delta t=T \sum_{j=0} E(j)$,

$\frac{d e(t)}{d t} \approx \frac{E(k)-E(k-1)}{\Delta t}=\frac{E(k)-E(k-1)}{T}$.

We can get proportional - integral - derivative formula:

$P(k)=K_{p}\left\{E(k)+\frac{T}{T_{I}} \sum_{j=0}^{k} E(j)+\frac{T_{D}}{T}[E(k)-\right.$

$-E(k-1)]\}$,

with $\Delta t=T$, which means the sampling period. When $T$ is small enough, the system is accurate. $E(k)$ refers to deviation value when sampling for the k's time; $E(k-1)$ refers to deviation value when sampling for the $(k-1)^{\prime} \mathrm{s}$ time; $k$ refers to the No. of sampling, $k=0,1,2, \ldots ; P(k)$ refers to the output when sampling for the k's time.

From $(31)$, to calculate $P(k), E(k)$ and $E(k-1)$ representing deviation signals of this time and last time are needed, and deviation signal $E(j)$ of every time should be added up together, that is, $\sum_{j=0}^{\mathrm{k}} E(j)$. This approach is complicated and needs much cache for storing $E(j)$, which is unbearable for DTN nodes. In DTN, it is not proper to control this way. According to recursive principle: proportional - integral - derivative formula for the $(k-1)$ time:

$P(k-1)=K_{p}\left\{E(k-1)+\frac{T}{T_{I}} \sum_{j=0}^{k-1} E(j)+\right.$

$\left.+\frac{T_{D}}{T}[E(k-1)-E(k-2)]\right\}$,

(31) minus (32) and here is the incremental formula:

$\Delta P(k)=P(k)-P(k-1)=K_{p}[E(k)-E(k-1)]+$
$+K_{I} E(k)+K_{D}[E(k)-2 E(k-1)+E(k-2)]$,
$K_{I}=K_{P} \frac{T}{T_{I}}$ refers to derivative coefficient, $K_{D}=K_{P} \frac{T}{T_{D}}$ refers to integral coefficient. As incremental deviation is related to deviation and the derivative is not easy to lose control, thus this research decides to adopt incremental proportional - integral - derivative formula. In real system, it is also necessary to consider limit problem, that is, the controller needs to be limited within a certain range, for example, probability $P$ needs to be limited between 0 and 1 , or adjust the upper limit $P_{\max }$ or the lower limit $P_{\min }$ in accordance with traffic degree to adapt to different networks.

\section{Discussion}

This research first introduces the characteristics of DTN routing, the classification of routing protocol and three indices to measure performance of routing algorithm: delivery probability, average delay and copy overhead. Then it analyses some of the common DTN routing protocols. Next, it introduces the aware of routing in DTN, in which community distribution, closeness, centrality, similarity and other selfish behaviours that are significant to the performance of routing. Social aware DTN routing is a focus of many researches [18, 26, 30, 36, 37].

With these awares to help with transmission, social aware routing algorithm can perform better than common DTN routing algorithm. The fruitful result of this research is that social aware DTN constructs a transmission strategy that is based on community distribution and centrality. This research adopts distributed K-Clique algorithm to conduct community distribution [35] and uses singlewindow centrality to calculate whole centrality and partial centrality. To control copy overhead, this research proposes a dichotomy approach. It is easy to operate and guarantees the copy overhead is within control (copy overhead won't exceed the original copy). This research proposes related message-weight function based on movement model of DTN and confirms opportunistic transmission and the priority of losing message in accordance with the function. Finally, this research adopts queuing management based on proportional - integral - derivative to avoid the overflow or internet traffic.

\section{Conclusion}

This research presents main ideas and focus of related routing algorithm by analysing social aware in DTN. It adopts distributed K-Clique algorithm to divide internet into different societies according to social aware in DTN [35]. To measure the significance of node in the internet and the community, this research adopts centrality as a comparative standard to help with routing transmission.

This research adopts simple and efficient dichotomy approach to reduce copy overhead. It constructs utility function for message with delivery probability as the target function. This utility function provides support for opportunistic transmission and message priority in proportional - integral - derivative queuing management.

It systematically solves main problems in DTN routing decision. Is it necessary to transmit message when the source node meets some other nodes? How to judge the 
importance of node in DTN? When to transmit message to relay nodes? How to confirm the priority of message in opportunistic transmission and queuing management? How to avoid the overflow or the internet traffic?

This research consists of three main parts:

1) It systematically introduces the background of DTN, its unique internet structure and the characteristics of DTN routing. It also states standards in DTN, namely, the definition of piles and their related format.

2) It discusses into the current situation of DTN. To begin with, it introduces the classification of DTN and performance standards. Then it introduces the current development of routing protocol though leaves out the background of DTN. Finally, it analyses social aware of DTN and social aware routing algorithm. It covers the most representative routing protocols.

3) It proposes social aware routing transmission strategy based on community distribution and centrality, and applies it to the proposed algorithm. This research adopts distributed d-clique algorithm as communitydiscovering algorithm with centrality as the most important standard to measure nodes. It proposes simple and efficient dichotomy approach to control copy overhead and construct utility function based on movement model of nodes. Finally, it adopts proportional - integral - derivative controller for queuing management.

\section{Reference}

[1] Zhang, Z. S. Routing in intermittently connected mobile ad hoc networks and delay tolerant networks: overview and challenges. // IEEE Communications Surveys \& Tutorials. $8,1(2006)$, pp. 24-37.

[2] Fall, K. A delay-tolerant network architecture for challenged internets. // Proceedings of the 2003 conference on Applications, technologies, architectures, and protocols for computer communications. ACM, pp. 27-34, 2003.

[3] Jain, S. et al. Using redundancy to cope with failures in a delay tolerant network. // ACM SIGCOMM Computer Communication Review. 35, 4(2005), pp. 123.

[4] Burleigh, S. et al. Delay-tolerant networking: an approach to interplanetary internet. // IEEE Communications Magazine. 41, 6(2003), pp. 128-136.

[5] Jones, Evan, P. C. et al. Practical routing in delay-tolerant networks. // IEEE Transactions on Mobile Computing. 6, 8(2007), pp. 943-959.

[6] Vahdat, A.; Becker, D. Epidemic routing for partially connected ad hoc networks. // Technical Report CS200006. Duke University, 2000.

[7] Lindgren, A.; Doria, A.; Schelén, O. Probabilistic routing in intermittently connected networks. // ACM SIGMOBILE Mobile Computing and Communications Review. 7, 3(2003), pp. 19-20.

[8] Spyropoulos, T.; Psounis, K. Raghavendra C S. Spray and wait: an efficient routing scheme for intermittently connected mobile networks. // Proceedings of the 2005 ACM SIGCOMM workshop on Delay-tolerant networking. (2005), pp. 252-259.

[9] Zhao, W.; Ammar, M.; Zegura, E. A message ferrying approach for data delivery in sparse mobile ad hoc networks. // Proceedings of the $5^{\text {th }} \mathrm{ACM}$ international symposium on Mobile ad hoc networking and computing. pp. 187-198, 2004.
[10] Jain, S.; Fall, K.; Patra, R. Routing in a delay tolerant network. // Proceedings of the ACM SIGCOMM Computer Communication Review. 34, 4(2004), pp. 145-157.

[11] Balasubramanian, A.; Levine, B.; Venkataramani, A. DTN routing as a resource allocation problem. // Proceedings of the ACM SIGCOMM Computer Communication Review. 37, 4(2007), pp. 373-384.

[12] Burgess, J.; Gallagher, B.; Jensen, D. et al. MaxProp: Routing for Vehicle-Based Disruption-Tolerant Networks. // Proceedings of the $25^{\text {th }}$ Conference on Computer Communications. IEEE, 6, (2006), pp. 1-11.

[13] Nelson, S. C.; Bakht, M.; Kravets, R. Encounter-based routing in DTNs. // Proceedings of the $28^{\text {th }}$ Conference on Computer Communications. IEEE, pp. 846-854, 2009.

[14] Liu, C.; Wu, J. An optimal probabilistic forwarding protocol in delay tolerant networks. // Proceedings of the $10^{\text {th }}$ ACM international symposium on Mobile ad hoc networking and computing. (2009), pp. 105-114.

[15] Zhu, Y.; Xu, B.; Shi, X. et al. A survey of social-based routing in delay tolerant networks: positive and negative social effects. // IEEE Communications Survey \& Tutorials. 15, 1(2013), pp. 387-401.

[16] Hui, P.; Crowcroft, J. How small labels create big improvements. // Proceedings of the $5^{\text {th }}$ Annual IEEE International Conference on Pervasive Computing and Communications Workshops. (2007), pp. 65-70.

[17] Hui, P.; Chaintreau, A.; Scott, J. et al. Pocket switched networks and human mobility in conference environments. // Proceedings of the 2005 ACM SIGCOMM workshop on Delay-tolerant networking. (2005), pp. 244-251.

[18] Pan, H.; Jon, C.; Eiko, Y. BUBBLE Rap: Social-Based Forwarding in Delay-Tolerant Networks. // IEEE Transactions on Mobile Computing. 10, 11(2011), pp. 1576-1589.

[19] Daly, E. M.; Haahr, M. Social network analysis for routing in disconnected delay-tolerant manets. // Proceedings of the $8^{\text {th }}$ ACM international symposium on Mobile ad hoc networking and computing. (2007), pp. 32-40.

[20] Bulut, E.; Szymanski, B. K. Friendship based routing in delay tolerant mobile social networks. // Proceedings of the 2010 IEEE Global Telecommunications Conference. (2010), pp. 1-5.

[21] Mei, A.; Morabito, G.; Santi, P. et al. Social-aware stateless forwarding in pocket switched networks. // Proceedings of the $30^{\text {th }}$ IEEE Conference on Computer Communications. (2011), pp. 251-255.

[22] Gao, W.; Cao, G. User-centric data dissemination in disruption tolerant networks. // Proceedings of the $30^{\text {th }}$ IEEE Conference on Computer Communications. pp. 3119 3127, 2011.

[23] Fabbri, F.; Verdone, R. A. Sociability-based routing scheme for delay-tolerant networks. // EURASIP Journal on Wireless Communications and Networking. 1(2011), pp. $1-13$.

[24] Rothfus, D.; Dunning, C.; Chen, X. Social-Similarity-based Routing Algorithm in Delay Tolerant Networks. // Proceedings of the 2013 IEEE International Conference on Communications. (2013), pp. 1-5.

[25] Wu, J.; Wang, Y. Social feature-based multi-path routing in delay tolerant networks. // Proceedings of the $31^{\text {st }}$ IEEE Conference on Computer Communications. (2012), pp. 1368-1376.

[26] Wang, Y.; Wu, J. Social-Tie-Based Information Dissemination in Mobile Opportunistic Social Networks. // Proceedings of the $14^{\text {th }}$ IEEE International Symposium on a World of Wireless, Mobile and Multimedia Networks. (2013), pp. 1-6.

[27] Mashhadi, A. J.; Mokhtar, S. B.; Capra, L. Habit: Leveraging human mobility and social network for efficient content dissemination in delay tolerant networks. // IEEE 
International Symposium on World of Wireless, Mobile and Multimedia Networks \& Workshops. 2009.

[28] Danon, L.; Diaz-Guilera, A.; Duch, J. et al. Comparing community structure identification. // Journal of Statistical Mechanics: Theory and Experiment. 09, (2005), pp. 1-10.

[29] Clauset, A. Finding local community structure in networks. // Physical review E. 72, 2(2005), pp. 1-6.

[30] Small, T.; Zygmunt, J. H. Resource and performance tradeoffs in delay-tolerant wireless networks. // Proceedings of the 2005 ACM SIGCOMM workshop on Delay-tolerant networking. 2005.

[31] Costa, P. et al. Socially-aware routing for publish-subscribe in delay-tolerant mobile ad hoc networks. // IEEE Selected Areas in Communications. 26, 5(2008), pp. 748-760.

[32] Hui, P.; Yoneki, E.; Chan, S. Y. et al. Distributed community detection in delay tolerant networks. // Proceedings of $2^{\text {nd }} \mathrm{ACM} / \mathrm{IEEE}$ international workshop on Mobility in the evolving internet architecture. (2007), pp. 1-8.

[33] Palla, G. et al. Uncovering the overlapping community structure of complex networks in nature and community. // Nature 435.7043, (2005), pp. 814-818.

[34] Spyropoulos, T.; Psounis, K.; Raghavendra, C. S. Performance analysis of mobility-assisted routing. // Proceedings of the $7^{\text {th }}$ ACM international symposium on Mobile ad hoc networking and computing. (2006), pp. 4960.

[35] Karagiannis, T.; Le Boudec, J. Y.; Vojnovic, M. Power law and exponential decay of intercontact times between mobile devices. // IEEE Transactions on Mobile Computing. 9, 10(2010), pp. 1377-1390.

[36] Elwhishi, A.; Ho, P.; Naik, K. et al. A Novel Message Scheduling Framework for Delay Tolerant Networks Routing. // IEEE Transactions on Parallel and Distributed Systems. 24, 5(2013), pp. 871-880.

[37] Gao, W. et al. Multicasting in delay tolerant networks: a social network perspective. // Proceedings of the tenth ACM international symposium on Mobile ad hoc networking and computing. 2009.

[38] Li, Q. H.; Zhu, S. C.; Cao, G. H. Routing in socially selfish delay tolerant networks. // IEEE INFOCOM. 2010.

\section{Authors' addresses}

Ying-Chiang Cho

Department of Electrical Engineering,

National Chung Cheng University,

Chia-Yi 62102, Taiwan

E-mail: silvergun@mail2000.com.tw 